\title{
El discurso antifeminista como recurso electoral en Brasil y España*
}

\section{Anti-feminist discourse as an electoral tool in Brazil and Spain}

Soledad Castillo Jara ${ }^{\star *}$

Pontificia Universidad Católica del Perú

ORCID: 0000-0003-0374-9730

Valerie Marchena Montalvo ${ }^{* *}$

Pontificia Universidad Católica del Perú

ORCID: 0000-0003-0419-9849

Sandra Quiliche Arévalo*

Pontificia Universidad Católica del Perú

ORCID: 0000-0001-9671-0266

Fecha de recepción: 16 de noviembre

Fecha de aceptación: 15 de diciembre

\section{ISSN: 2415-2498}

Castillo Jara, S., Marchena Montalvo, V., Quiliche Arévalo, S. (2019). El discurso antifeminista como recurso electoral en Brasil y España. Politai: Revista de Ciencia Política, Año 10, Nº19, pp. 35-59.

DOI: https://doi.org/10.18800/politai.201902.002

\footnotetext{
* Las autoras agradecen la colaboración de José Arturo Tipismana Arriola en la elaboración del marco teórico y de Carol Inga Correa en la revisión bibliográfica. Ambos son estudiantes de Ciencia Política y Gobierno de la Pontificia Universidad Católica del Perú (PUCP).

** Licenciada en Ciencia Política y Gobierno por la Pontificia Universidad Católica del Perú (PUCP). Asistente de investigación en el Centro de Investigación de la Universidad del Pacífico (CIUP) y pre docente del curso de Relaciones Internacionales en la Facultad de Ciencias Sociales de la PUCP.

*** Estudiante de sexto ciclo de Ciencia Política y Gobierno en la Pontificia Universidad Católica del Perú (PUCP).

**** Estudiante de noveno ciclo de Ciencia Política y Gobierno en la Pontificia Universidad Católica del Perú (PUCP) y asistente de investigación en el Centro de Estudios Financieros (CEFI) de la Asociación de Bancos del Perú.
} 


\section{RESUMEN}

El presente artículo se centra en dos casos correspondientes a partidos y candidatos que se ubican más a la derecha de lo habitual y, por ello, han sido calificados como de extrema derecha. Estos son Jair Bolsonaro, quien fue candidato del Partido Social Liberal y actualmente es presidente de Brasil luego de haber ganado en la segunda ronda de las elecciones generales del 7 de octubre de 2018 con el 55,1\% de los votos; y el partido Vox, que logró ingresar por primera vez al parlamento nacional en España luego de las elecciones generales del 28 de abril de 2019 y es actualmente la tercera fuerza parlamentaria con el $15,09 \%$ de los votos y 52 escaños.

Para ambos, se propone estudiar el uso de un discurso político antifeminista como recurso electoral en sus campañas. Pese a las diferencias en los sistemas electorales y en los diseños institucionales de ambos países -uno presidencial y otro parlamentario- ambos casos comparten el hecho de que las candidaturas calificadas como de extrema derecha han logrado convencer a los electores, aunque en distinto grado, apelando al descontento en contra de las ideas y políticas progresistas de los anteriores gobiernos de izquierda, el del Partido de los Trabajadores en Brasil (PT) y el del Partido Socialista (PSOE) en España. Entre dichas ideas se encuentra, evidentemente, la lucha por la igualdad de género que es calificada por los partidos de extrema derecha como una "ideología de género". Concluimos que, si bien ambos hacen uso de un discurso reñido con las posturas feministas, el cuestionamiento que presentan no ataca a todo el conjunto de ideas de dicho movimiento, sino que se dirige contra las más contemporáneas o post-materiales (como el sistema de cuotas o el lenguaje inclusivo), mientras que reconoce e incluso elogia los logros más clásicos o materiales del feminismo (como la independencia económica de las mujeres). Asimismo, se encuentra una complejidad mayor en el debate en el caso español, lo cual nos lleva a pensar que el cuestionamiento planteado por Vox puede servir para repensar las ideas y políticas progresistas de los últimos gobiernos de una manera más general, que trasciende el ámbito del tema de género.

Palabras clave: feminismo, discurso anti-feminista, valores post-materiales, extrema derecha, Brasil, España. 


\section{Abstract}

This paper focuses on two cases corresponding to parties and candidates situated more to the right than usual and, therefore, described as far right. These cases are Jair Bolsonaro, former candidate of the Social Liberal Party and current president of Brazil after winning in the second round of the general elections of October 7, 2018 with 55.1\% of the votes; and Vox, the party which achieved parliamentary representation in Spain for the first time after the general elections of April 28, 2019 and is currently the third parliamentary force with $15,09 \%$ of the votes and 52 seats.

For both, we propose to study the use of anti-feminist political discourse as an electoral tool. In spite of the differences in the electoral systems and in the institutional designs of both countries - one presidential and the other parliamentary - both cases share the fact that the candidates have managed to convince the electors, although in different degrees, appealing to discontent against the progressive ideas and policies of the previous leftist governments, that of the Workers Party (PT) in Brazil and that of the Socialist Party (PSOE) in Spain. Evidently, among these ideas is the struggle for gender equality that is described by the extreme right parties as a "gender ideology." We conclude that, although both use a discourse which is at odds with feminism, the questioning they present does not attack the whole set of ideas of that movement. Instead, it is directed against the most contemporary or post-material ones (such as the quota system or inclusive language), while recognizing and even praising the most classic or material achievements of feminism (such as women's economic independence). Additionally, there is a greater complexity in the debate in Spain, which leads us to think that the questioning raised by Vox may be useful to rethink the progressive ideas and policies of the last governments in a more general way, which transcends the scope of gender.

Keywords: feminism, anti-feminist discourse, post-material values, far right, Brazil, Spain. 


\section{INTRODUCCIÓN}

El mundo actual atraviesa un periodo de notable polarización política. A lo largo de los últimos años, partidos y candidatos ubicados más a la izquierda o más a la derecha de aquellos que habitualmente gobernaban han conseguido victorias de diversa magnitud. Algunos lograron gobernar — por ejemplo, por el lado de la izquierda, el partido Syriza en Grecia y, por el lado de la derecha, Donald Trump en Estados Unidos. Otros estuvieron muy cerca de lograrlo - como el Frente Nacional francés, cuya candidata Marine Le Pen pasó a la segunda ronda de las elecciones presidenciales de 2017 con el 21,30\% de los votos. Y otros, sobre todo en sistemas parlamentarios, pese a no formar parte del gobierno, lograron influir en la agenda de este al posicionar en el debate público los temas que venían defendiendo desde sus campañas — este es el caso, por ejemplo, de Alternativa para Alemania en relación al tema de la multiculturalidad y la gestión migratoria.

Esta polarización política que caracteriza a nuestro tiempo responde a múltiples motivaciones. Por un lado, existen factores materiales como el incremento de la desigualdad, la precariedad de los empleos o el deterioro de la calidad de vida, que en Europa y Estados Unidos reflejan las consecuencias de la crisis económica del año 2008, mientras que en América Latina están vinculados a las políticas de ajuste estructural llevadas a cabo desde los años noventa. Por otro lado, sin embargo, el descontento social que alimenta la polarización política también tiene un importante componente de ideas.

En ambos casos de estudio, el componente ideacional se basa en una dinámica de acción y reacción alrededor de las ideas progresistas que varios Estados han venido incorporando y aplicando en los últimos años respecto a temas sensibles como la desigualdad de género, la migración, la multiculturalidad, la laicidad, los derechos de las personas LGTB, entre otros. Mientras que los sectores de izquierda buscan el fortalecimiento de este tipo de ideas, y los gobiernos — con mayor o menor grado de compromiso - apoyan su continuidad, han empezado a surgir con mayor fuerza corrientes de derecha que perciben en ello una peligrosa imposición de lo políticamente correcto. Tal como se plantea en el texto de Fukuyama (2018), las demandas que sustentan la polarización política actual no solo han de ser satisfechas a través de medios económicos, sino que hay también un fuerte componente de demandas de reconocimiento. En el caso de los partidos y candidatos más conservadores, como los que analizaremos en este texto, la demanda es por el reconocimiento de sus ideas como planteamientos igualmente válidos y no inferiores a los planteamientos progresistas.

Ante este escenario de polarización, surge la importancia de estudiar a aquellos partidos y candidatos que se ubican en los extremos del espectro político. Actualmente, dada la preocupación y la polémica que generan a nivel internacional los partidos y candidatos calificados como de extrema derecha, consideramos que es particularmente necesario entender sus discursos con mayor profundidad. Seleccionamos los casos de Jair Bolsonaro en Brasil y Vox en España. Admitimos que estos casos presentan notables diferencias; en primer lugar, en un caso nos referimos a un candidato y en el otro, a un partido dado que los sistemas políticos de ambos países son distintos; asimismo, en un caso tenemos al vencedor de la contienda electoral nacional y en el otro, al partido que 
representa la tercera fuerza política en el Congreso de los Diputados pero no gobierna es más, hasta el momento de escribirse este artículo aún no hay un Presidente del Gobierno investido en España. Sin embargo, consideramos que es importante compararlos ya que ambos surgen como reacción a gobiernos progresistas previos que trabajaron para superar algunos desafíos relacionados a la desigualdad de género, por ejemplo, en temas de prevención y sanción de la violencia o en cuanto a las cuotas de mujeres en puestos de representación política. Pero ahora son precisamente estos aspectos, que podrían ser vistos como avances de los gobiernos anteriores, los que resultan cuestionados por las candidaturas calificadas como de extrema derecha.

A continuación, se presentará primero un marco teórico sobre los discursos de los partidos de extrema derecha, en el cual se identificará algunas características centrales de los mismos. Luego, se procederá al estudio de cada caso en tres partes: una descripción general del partido o candidatura que estamos analizando, una reseña de los avances que ha conseguido el feminismo en cada país — principalmente desde la segunda mitad del siglo $\mathrm{XX}$ - y finalmente un análisis del discurso actual del partido o candidato en cuestión con énfasis en la problemática de género. Para esta última sección, la metodología consistirá en la recopilación y análisis de fuentes secundarias de diverso tipo, tanto bibliográficas como de redes sociales (Twitter y Facebook) y material audiovisual (vídeos publicados por el propio partido o candidato y entrevistas en medios de comunicación). La revisión de perfiles en redes sociales y vídeos fue hecha a lo largo de todo el año 2019, con énfasis en fechas relevantes como el 8 de marzo (Día Internacional de la Mujer) y el 25 de noviembre (Día Internacional de la Eliminación de la Violencia contra la Mujer). Asimismo, en ambos casos, el objeto de análisis no se limitará al candidato principal, es decir Jair Bolsonaro o Santiago Abascal, sino que también abarcará a otros personajes relevantes, ya sea por su jerarquía dentro del partido o por su vinculación específica con el tema de género. Serán centrales Damares Alves en el caso de Brasil y Rocío Monasterio en el caso de España.

\section{MARCo Té́rico: El DISCURSO DE LA EXTREMA DERECHA O DERECHA RADICAL}

Según Cas Mudde (2017), los partidos de derecha radical populista comparten 3 características principales: el nativismo, el autoritarismo y el populismo. La primera característica hace referencia al versus entre nativos y foráneos, generando discriminación hacia los extranjeros, dentro de un discurso de la otredad o de endogrupoexogrupo (nosotros vs ellos). El nativismo es una variante de nacionalismo étnico, es una ideología que sostiene que los Estados deberían ser habitados exclusivamente por miembros del grupo nativo (la nación) y que los elementos no-nativos (personas e ideas foráneas) son fundamentalmente una amenaza para un Estado-nación homogéneo» (Mudde, 2007: 19).

La segunda característica se manifiesta en la invocación que hacen estos partidos a someterse a las normas y a la autoridad, es decir, respetan el juego democrático, pero tratan de llegar al poder para subvertirlo. Esto se refleja también en la obediencia rígida de los militantes y simpatizantes hacia los líderes partidarios. Finalmente, la tercera característica se observa cuando estos partidos se autodenominan como los únicos 
representantes del pueblo, en contraposición a un gobierno de élites progresistas que no representa los verdaderos intereses de la población; esto remarca también su carácter nopluralista, pues desconoce la legitimidad de las demás propuestas políticas.

Taguieff (2007) apunta que estos partidos de derecha radical son una forma nueva y específica de populismo. De acuerdo con este autor, si el populismo político clásico se basaba en una apelación a las clases populares en confrontación con una clase política corrupta y ensimismada, en el discurso de estos nuevos movimientos se apela a una comunidad nacional interclasista, frente a un enemigo externo (los inmigrantes) y otro interno (los políticos).

Para situar este ascenso de la derecha radical en un contexto global, Norris e Inglehart (2017) explican que, tras el auge de los valores post-materiales, el apoyo a los partidos xenófobos y radicales tiene una explicación distinta al ascenso de estos mismos en los años recientes: creen que el backlash cultural explica por qué hay votantes que apoyan a los partidos xenófobos; pero que el declive económico y el riesgo a la seguridad son los factores que ayudan a explicar por qué esos partidos son más fuertes hoy. Consideran que, el que la discusión política se haya situado en el plano post-material, hace que los temas de campaña sean precisamente cuestiones post-materiales, lo que refuerza el hermetismo de aquellos que están en desacuerdo con las banderas progresistas. Esto es precisamente lo que se evidencia en los casos que estudiamos, un discurso que se opone a los aspectos post-materiales del feminismo en cada país.

Por su parte, Farid Kahhat (2019), sistematiza en dos grandes vertientes, los estudios realizados sobre la derecha radical: (1) el análisis de la demanda, es decir, qué condiciones estructurales contribuyen a entender el fenómeno, sean económicas o culturales, qué es lo que motiva a los votantes a optar por esta alternativa política. (2) el análisis de la oferta, es decir, explica la influencia sobre los votantes y la agenda política que ejercen los partidos y movimientos de derecha radical con base en sus características internas, su conducta política y las oportunidades que ofrece el diseño institucional. En esa línea, el presente artículo se encuentra en la segunda vertiente de los estudios de la derecha radical.

Finalmente, entendemos que el marco teórico antes presentado cumple un papel importante para explicar la extrema derecha dentro del espectro político, pero a la vez reconocemos que fue originalmente pensado para contextos como los de Europa y Estados Unidos. Por lo tanto, encuentra sus limitaciones en casos latinoamericanos como el de Brasil. Ello es entendible también considerando la reciente anomalía dentro de la política latinoamericana que ha significado la llegada al poder de un personaje como Bolsonaro.

\section{El CASO DE BRASIL}

Jair Bolsonaro obtuvo la presidencia de Brasil el 7 de octubre de 2018, luego de haber postulado como candidato del Partido Social Liberal. Este fue un partido de derecha fundado durante la década de los 90. Previamente a las elecciones del 2018, solía tener un rendimiento electoral pobre. Si bien el partido compartía determinados principios 
ideológicos con el candidato, fue Bolsonaro quien, mediante estrategias populistas y discursos de otredad, logró concentrar alrededor de su figura la resistencia de la población conservadora de Brasil en contra de las políticas de tintes post-materialistas del anterior gobierno. El ahora presidente estuvo constantemente en la polémica nacional e internacional por sus comentarios sobre los derechos de las mujeres y las personas LGBT, la izquierda y la dictadura militar brasileña, lo cual le valió el calificativo de candidato de extrema derecha.

Uno de los factores más importantes para explicar por qué Bolsonaro llegó al poder es la pérdida de legitimidad del gobierno de izquierda liderado por el Partido de los Trabajadores (PT). El PT, primero con Lula Da Silva (2003-2010) y luego con Dilma Rousseff (2011-2016), gobernó el país a través de la presidencia y de gobiernos de coaliciones de mayoría en el Parlamento, aliándose principalmente con partidos de izquierda. Tras los escándalos de corrupción generados por el caso Lava Jato, que involucra a la empresa Odebrecht y los altos mandos de dicho partido, el PT quedó profundamente desprestigiado.

Asimismo, para explicar por qué se produjo este aparente giro a la derecha en Brasil, es necesario repasar brevemente el último gobierno de Rousseff. Tanto los dos gobiernos de Lula da Silva como el primer gobierno de Dilma Rousseff se caracterizaron por la expansión del Estado orientado a políticas redistributivas y de bienestar social, siguiendo la tendencia del llamado «giro a la izquierda» en América Latina (Levitsky y Roberts, 2011: 16). Debido al gasto público que implica, esta orientación del Estado se sustentó sobre un panorama económico favorable; pero este se vio opacado durante el segundo gobierno de Rousseff debido que la economía entró en recesión. Desde antes de la reelección de la mandataria ya se advertía una desaceleración en el crecimiento económico del país; sin embargo, este se encontraba en su fase inicial y aún no era relacionado con la gestión de la presidenta (Grugel y Riggirozzi, 2018).

Jair Bolsonaro, desde su discurso inaugural del 1 de enero de 2019, prometió una serie cambios políticos y económicos para Brasil; pero, a la vez, vinculó estas propuestas con su agenda profundamente conservadora. Prometió «liberar definitivamente al país del yugo de la corrupción, de la violencia y de la sumisión ideológica» y posteriormente expresó sobre la «necesidad de erradicar el socialismo y la ideología de género» (Bolsonaro, 1 de enero de 2019). Ello evidenció el giro conservador del gobierno central y la nueva visión que el mandatario tiene del país y de su pueblo. Poco tiempo después de este discurso, Brasil regresó al debate internacional debido a las declaraciones de la ministra de la Mujer, la Familia y los Derechos Humanos, Damares Alves, quien comentó que en el gobierno de Bolsonaro se iniciaría una nueva era en la que «los niños visten de azul y las niñas de rosa» (Alves, 2 de enero de 2019).

\subsection{Revisión contextual sobre el feminismo en Brasil}

América Latina tuvo un movimiento feminista destacado desde finales del siglo XIX hasta las primeras décadas del XX. La lucha por la ciudadanía fue moldeada en gran medida por la experiencia colonial y la historia política de las democracias en crisis 
(Molyneaux, 2003). Durante el gobierno de Getulio Vargas y con la llegada de nuevos paradigmas feministas provenientes de otras partes del mundo, hubo una fragmentación de las posturas esgrimidas por los movimientos feministas entre la visión de la mujer como ángel del hogar y una visión mucho más vanguardista que identifica a la misma como sujeto con capacidad de emancipación.

Durante los años 60, los movimientos de mujeres se fragmentaron en la búsqueda de igualdad práctica y políticas públicas que buscaran reducir las brechas de género enmarcadas en el periodo reformista y nacionalista brasileño que finaliza en 1964 con la llegada al poder del presidente Castelo Branco y que fueron reacción a la restricciones los derechos políticos a las miembros del Movimiento Femenino por la Amnistía para denunciar la violación sistemática de los derechos humanos por parte de la dictadura brasileña. En contraste con ello, existió una reacción conservadora que buscaba mantener el orden representado por la familia tradicional, la propiedad privada, la sumisión a las instituciones formales, la moral religiosa y el rol protector de las fuerzas armadas (Blay, 1987). Suely y Brabo (2015) señalan que, durante los años 70, la herramienta para visibilizar y criticar la situación femenina fueron los estudios académicos desde distintas disciplinas. Dentro de este movimiento femenino vale recalcar el liderazgo e innovación tanto académica como políticamente de Eva Alternan Blay. Ella fundó el Centro de Estudios de Género y de los Derechos de la Mujer de la Universidad de São Paulo, el cual fue un instituto pionero en temas de género. En su rol activista, también busco el reconocimiento del trabajo de las mujeres en la academia (Suely y Brabo, 2015).

Durante los años 80, el movimiento feminista tuvo un cariz político en el cual las mujeres ya eran personas instruidas que buscaban el poder para lograr una serie de reformas para acabar con la discriminación en todos los ámbitos de la vida en sociedad. Por lo tanto, se buscó la creación de organismos que velen por la seguridad de más mujeres frente a temas como la violencia física y sexual, el acceso inequitativo a la política y la desigualdad económica. En estos años hubo un énfasis participativo que buscó la colaboración de la mayor cantidad de mujeres posible para implementar las políticas por la que antes habían salido a las calles (Borba, 1998). Se consiguió la creación del Consejo Nacional de los Derechos de la Mujer (1985) y la posterior Constitución de 1988. Asimismo, la asistencia a las mujeres en condiciones vulnerables o en posible riesgo se articuló desde los sindicatos, las ONG y los partidos políticos; estas instituciones brindaron desde asistencia psicológica y educativa hasta asistencia legal (Suely y Brabo, 2015).

La Constitución de 1988 evidenció que ya se tenía un análisis interseccional de la realidad femenina que enriqueció mucho el debate sobre los derechos y deberes femeninos. El nuevo ordenamiento legal empezó a reconceptualizar la familia tradicional mediante la abolición de la figura de jefe de familia. Se implementó también el divorcio y la licencia de maternidad extendida. Con el fin de equilibrar los roles de cuidado entre el hombre y la mujer, se crearon la licencia de paternidad y el derecho a la guardería. También se penalizó todo tipo de discriminación contra las mujeres en el ámbito laboral en general. Sin embargo, el giro neoliberal de la década siguiente afectó los logros 
obtenidos por los movimientos feministas e impidió una aplicación cabal de la carta magna. A finales de los 90, estas normas fueron insertadas solo tímidamente en la agenda política nacional; no hubo una correspondencia entre las ideas clave de la Constitución y la agendación de políticas públicas (Suely y Brabo, 2015).

Los años 2000 significaron la reactivación del movimiento feminista brasileño. Este buscó nuevas plataformas para insertar a más mujeres en la toma de decisiones y fortalecer la democracia a partir de feminismo e igualdad social sin importar condición económica, raza, etnia o similares. Hubo dos momentos cumbre en esta década: la creación de Secretaría Nacional de los Derechos de la Mujer (2003) y la Ley María da Penha (2006) que cambió el Código Penal para permitir que quienes agreden a mujeres y son capturados en flagrancia cuenten con detención preventiva, asimismo se endurecieron las sanciones a los victimarios y se establecieron mayores medidas de protección para las víctimas y sus hijos e hijas (Suely y Brabo, 2015).

Los gobiernos del PT establecieron una serie de políticas públicas para reducir las desigualdades sociales. Los mecanismos usados fueron asistencia económica través del programa Bolsa Família, el Programa Nacional para el Acceso a la Educación Técnica y Empleo (PRONATEC), la enseñanza sobre temas de género y derechos sexuales, entre otros (Palermo y Menezes, 2013). Este tipo de políticas se enfocaron en lograr la salida de la pobreza de muchas familias, en especial de mujeres en condiciones de riesgo, ya que la pobreza suele afectar de una manera más grave a la población femenina. Sin embargo, la deslegitimación de las posturas progresistas representadas por el PT generó un ambiente propicio para el avance de actores conservadores que nunca dejaron de ser parte del escenario político y aprovecharon momentos claves como este para recuperar su poder.

Entre estos actores conservadores de la política brasileña se encuentran los grupos evangélicos. Sus objetivos se visibilizan, por ejemplo, en la propuesta de reforma del Currículo Nacional, donde buscaron cuestionar la teoría de la evolución, demonizar a los homosexuales o describirlos como enfermos mentales, criminalizar el aborto sin excepciones, entre otros. El crecimiento de la fuerza política de los evangélicos, que juega un papel muy importante en la política brasileña, surge por su capacidad para apoyar o convertirse en políticos de distinto signo. Así, su apoyo ha virado en direcciones políticas muy variadas desde el respaldo a Lula Da Silva (Gómez, 2015) hasta su influencia en la llegada al poder de Bolsonaro. Tal como señalan Mariano y Gerardi (2019), con el avance electoral del PT a mediados de septiembre, trece iglesias evangélicas se convirtieron en bastiones opositores al PT y los pastores ocuparon las redes sociales para demonizar a los gobiernos petistas, al mismo partido y a su candidato Fernando Haddad. Por ejemplo, Silas Malafaia, de la Asamblea de Dios Vitória em Cristo, apuntó su «cañón digital» contra el PT y lo acusó de ser «el autor del kit gay», «esa cartilla del infierno para destruir a nuestros hijos» $\mathrm{y}$ «de estar a favor de erotizar a los niños en las escuelas» (Mariano y Gerardi, 2019: 69). 


\subsection{Ideario y discurso de Jair Bolsonaro}

El discurso de Bolsonaro recuerda a la dictadura militar que ha defendido férreamente cuatro pilares fundamentales: Fuerzas Armadas, familia tradicional, libre mercado e Iglesia. Tanto en sus presentaciones públicas como en sus redes sociales, Bolsonaro caracteriza al PT y a los partidos tradicionales brasileños como causantes de la pérdida de la moral. Los acusa por el predominio de la llamada "ideología de género" y por la crisis económica, política y social que aqueja al país. En su discurso de asunción de mando, Jair Bolsonaro señaló lo siguiente:

Estoy ante la nación entera en este día como el día en que la gente comenzó a liberarse del socialismo, la inversión de valores, el gigantismo estatal y la corrección política (...). No podemos permitir que ideologías dañinas dividan a los brasileños. Ideologías que destruyen nuestros valores y tradiciones, destruyen a nuestras familias, la base de nuestra sociedad (...). E invito a todos a comenzar un movimiento en esta dirección. Nosotros, usted y nuestras familias, todos juntos, podemos restablecer los estándares éticos y morales que transformarán nuestro Brasil (...).Tenemos el gran desafío de abordar los efectos de la crisis económica, el desempleo récord, la ideologización de nuestros hijos, la distorsión de los derechos humanos y la deconstrucción de la familia (...)Nuestra preocupación será la seguridad de las buenas personas y la garantía de los derechos de propiedad y la defensa propia, y nuestro compromiso es valorar y apoyar el trabajo de todas las fuerzas de seguridad (...) Brasil sobre todo, Dios sobre todo (Bolsonaro, 1 de enero de 2019).

Como se observa en la cita anterior, Bolsonaro no se expresa de forma explícita sobre los temas de género, sino que prefiere enmarcarlos dentro de un todo ideológico conformado por los preceptos progresistas en temas como la economía, la educación, la religión, el rol de las fuerzas armadas, el medio ambiente, los derechos indígenas, entre otros. Engloba y presenta a todos estos temas como la "herencia petista" y, por lo tanto, como representación del descalabro político, social y económico.

Por otro lado, el 2 de enero de 2019 se hizo público un video en el que la nueva ministra Alves saludaba a sus seguidores y demostraba cuál sería el enfoque de su trabajo en esta cartera: "Es una nueva era en Brasil: el niño viste de azul y la niña viste de rosa" (Alves, 2 de enero de 2019). Poco tiempo después, la ministra Alves señaló que ese comentario no era textual, sino que hacía referencia a que ella buscaba revertir la "ideología de género" que estaba impregnada en la educación brasileña.

Sin embargo, dentro del perfil oficial del Ministerio, publicó un mensaje en el que señaló que la estructura del ministerio pasaría a tener ahora ocho secretarías nacionales es decir, dos más que en la administración anterior. Estas serían la de Familia y la de Juventud. Asimismo, destacó que la actual Dirección para la Promoción de los Derechos de las Lesbianas, Gays, Bisexuales, Travestis y Transgénero, anteriormente un organismo de la Secretaría Nacional de Ciudadanía, se mantendría, con la misma estructura, dentro de la Secretaría Nacional de Protección Global (Ministério da Mulher, da Família e dos Direitos Humanos, 2019). Este mensaje oficial fue, de cierto modo, contradictorio con la idea de oponerse a los anteriores avances en políticas de género y también al mensaje de Bolsonaro respecto al llamado "gigantismo estatal". 
El 22 de enero, luego de ser criticada por medios nacionales y extranjeros, Alves intenta explicar cuál es el enfoque de derechos humanos que defenderá y por el cual trabajará su cartera: "Defenderemos la familia y los verdaderos derechos humanos; proteger el derecho a la vida y a la propiedad privada y promover una educación que prepare a nuestros jóvenes para los desafíos de la cuarta revolución industrial reduciendo a sabiendas la pobreza y la miseria" (Alves, 22 de enero de 2019). Sin embargo, el día 29 de enero se hace pública su reunión con ONU mujeres donde comenta:

Ayer recibí una visita de ONU Mujeres y, además de conocer propuestas muy interesantes en la lucha contra la violencia contra las mujeres, también les pedí que participaran e investigaran para traer a Brasil experiencias exitosas en la lucha contra el suicidio y la automutilación de mujeres (Alves, 29 de enero de 2019).

Entonces sí se evidencia el interés de la ministra para combatir la violencia contra las mujeres, pero se evita usar en el mensaje palabras como "género" o "machismo". Se construye el problema de la violencia como un asunto general, sin incluir un tratamiento especial por el hecho de que la víctima sea una mujer. Asimismo, se visibilizan otras problemáticas diferentes a la lectura tradicional de las políticas de género, como el suicidio y la automutilación. Este discurso de no distinción entre poblaciones en el tema de la violencia es reafirmado en junio, cuando Alves señala en Twitter que "este es un gobierno que trabaja para erradicar todo tipo de violencia, ya sea contra mujeres, hombres, LGBTI +, niños, adolescentes, ancianos,..." y añade el hashtag \#DerechosHumanosParaTodos.

El 18 de febrero se publicó una entrevista en la cual la ministra Alves explicó su postura respecto al feminismo y a las personas homosexuales:

Sería exagerado hablar de anti-activismo. Hay pautas feministas que abrazo. Por ejemplo, la igualdad salarial para hombres y mujeres y la lucha contra la violencia. Si es necesario para mí y para las feministas salir de la mano a la calle, lo haré. Pero sin la exageración de los senos en exhibición. Sin el adoctrinamiento que parece predicar el odio a los hombres (Alves, citada por Ohara, 18 de febrero de 2019)

Tengo una posición muy clara sobre esto y el movimiento LGBT lo sabe. Si sale todo como ellos quieren, los cristianos pueden ser criminalizados por no aceptarlo. Porque ellos entienden que cuando los cristianos dicen que la práctica de la homosexualidad es un pecado, puede haber discriminación. No es discriminación, es una expresión de fe. Respeto a los hombres homosexuales y no tengo nada en contra de su elección sexual. Pero hay movimientos que han utilizado a los homosexuales al servicio de los partidos políticos y la ideología de género. Eso es lo que debemos revisar (Alves, citada por Ohara, 18 de febrero de 2019).

En el mes de abril, la ministra Alves volvió a enmarcar un tema reñido con el feminismo dentro de la defensa de la libertad religiosa. En esta oportunidad, afirmó en la Cámara de Diputados que "en la concepción cristiana la mujer debe ser sumisa al hombre en el matrimonio" (Alves, 16 de abril de 2019). Desde su perfil personal, el 16 de ese mismo mes, ella explicó este comentario de la siguiente manera:

Tenga en cuenta que no estoy abogando por que todos sigan este punto de vista. Es una cuestión de libertad religiosa, garantizada por la Constitución. No creas las mentiras que dices al respecto. Las mujeres que son víctimas de violencia nunca deben ser sumisas. 
Deben denunciar al agresor. Una mujer cristiana, o de cualquier otra designación, que sea víctima de violencia debe denunciar a su abusador. Los líderes religiosos juegan un papel fundamental en el apoyo espiritual. Pero también para guiar e incluso acompañar a la mujer maltratada o amenazada (Alves, 16 de abril de 2019).

Entonces, cuando se habla de feminismo, ella reconoce que no es antifeminista, como sí se ha definido Bolsonaro en muchas ocasiones. Ha reconocido la importancia del movimiento y la existencia de frente comunes, por lo que existe una aprobación parcial. En términos del marco teórico que hemos presentado, diríamos que ella apoya el aspecto material del feminismo, que es el más antiguo en el tiempo y comprende demandas principalmente de seguridad personal y económicas. Sin embargo, se opone a la deriva post-material que ha tomado el feminismo en la actualidad para abarcar no solo nuevos temas sino también nuevas formas de manifestarse, como por ejemplo las protestas que la ministra menciona en las que las mujeres muestran los senos.

En cuanto a las personas homosexuales, la ministra enmarca su posición de rechazo a ellas dentro de la "libertad religiosa" de ciertos fieles para llamar pecado a una "elección", es decir, no ve este rechazo como homofobia o como una conducta que debería ser penalizada, sino que se ampara en la libertad de las personas para creer que la homosexualidad es pecado si así lo entienden según sus creencias religiosas. Asimismo, se refiere a la orientación sexual como una elección. Sin embargo, dentro del debate actual en temas de género ya no se usa el término “elección”, pues este daría a entender que uno elige cierto tipo atracción sexual y puede modificarlo a voluntad. Este es un término desfasado y ciertas comunidades lo consideran incluso insultante. Entonces, una vez más, se entiende que la postura de la ministra está alejada de las actuales derivas del movimiento feminista y LGTB.

El 25 de febrero, Alves publicó en su Twitter sobre cuál sería la "postura del ministerio" sobre la problemática del aborto: "Informamos al mundo que Brasil es un país nuevo y que defenderemos la vida desde la concepción” (Alves, 25 de febrero de 2019) y cita una noticia de $O$ Globo donde amplía la información y presenta el discurso de presentación de Brasil en la Comisión de Derechos Humanos de la ONU.

Siguiendo los posts oficiales del Ministerio, encontramos un énfasis en la defensa de la primera infancia, a partir de una protección a los menores contra los delitos sexuales y el "turismo sexual". Esto se materializó el 27 de febrero en el lanzamiento de la campaña "Mi cuerpo no es su fantasía" en marco de las celebraciones del Carnaval de Río de Janeiro.

El 12 de marzo, la ministra Alves se reúne con el Fondo de Población de Naciones Unidas, uno de los organismos más dedicados a temas de planificación familiar y derechos sexuales:

Recientemente hablé con la directora ejecutiva del Fondo de Población de las Naciones

Unidas, Natalia Kanem, y nos hemos comprometido a cooperar en cuestiones como la lucha contra las autolesiones, la desaparición de niños y el problema de los refugiados (Alves, 12 de marzo de 2019) 
Sin embargo, en el presente tuit se dejan en claro cuáles temas son tratados y, como si esto no fuera suficiente, horas después en el mismo tuit se agrega una precisión: "Este es un ministerio que defiende la vida (Alves, 12 de marzo de 2019)".

Siguiendo las recomendaciones de ONU, la ministra confirma que se darán una serie de reformas en la forma en la que se atienden a las víctimas de "violencia doméstica"-en ningún momento habla de violencia de género: "La mujer necesita cuidados especiales cuando está en el papel de víctima. La experiencia de denunciar la violencia doméstica ya no puede ser una experiencia traumática. Miremos la red de servicio para ver dónde está el error. Tenemos urgencia”. (Alves, 20 de marzo de 2019).

Sin embargo, el 27 de marzo se hizo público que esta cartera sería sede del evento "Trampas del feminismo", donde se presentaría el libro de la diputada del Partido Social Liberal Ana Caroline Campagnolo Feminismo: perversión y subversión. Según el ministerio, esto se hizo en el marco del "mes de la mujer". Cuando fue consultada sobre este polémico evento, la ministra Alves volvió a indicar que no es anti feminista ni nada similar, sino que las divergencias existen y que hay facciones del feminismo que ella no considera positivas; al mismo tiempo, añadió un elemento adicional: la necesidad de representatividad del bloque conservador de mujeres que, como ella, desean poner sus visiones en agenda (Alves, 27 de marzo de 2019).

Finalmente, si bien queda mucho por estudiar aún sobre el impacto de la llegada de Bolsonaro en las políticas públicas brasileña, podemos evidenciar que existe un desfase entre este y el actuar del ministerio liderado por Damares Alves. Desde el análisis de los documentos y publicaciones de dicho ministerio en su página web y redes sociales, se observa que, si bien existe un discurso y un liderazgo conservador, estos no han llegado a materializarse del todo en las políticas, ya que actúan en medio de una burocracia estatal marcadamente progresista. El gobierno reconoce la inconveniencia de una confrontación directa con los sectores progresistas que siguen teniendo un rol importante en la política brasileña. Asimismo, la postura abierta al diálogo de la ministra difiere mucho de los discursos de Bolsonaro. Ello no significa que el gobierno no haya actuado para desmantelar la herencia del PT; este ha sido su objetivo desde la candidatura, sino que por motivos prácticos ha terminado dándole un giro al ministerio hacia la niñez y la salud mental, temas que políticamente son menos controversiales.

Dentro del discurso oficial, se entiende a la violencia contra las mujeres y los crímenes contra la comunidad LGTBI como problemas públicos reales y que necesitan de acción estatal, lo que consideramos importante para el devenir de los derechos humanos en Brasil. Pero al mismo tiempo, reconocemos que existe una nueva perspectiva y atención desigual hacia ambas temáticas, lo cual ha significado la visibilización de problemas igualmente importantes como la automutilación, la violencia hacia la infancia y el suicidio. Estas nuevas perspectivas han significado también la censura de ciertas palabras como "género", "crímenes de odio" u "orientación sexual", lo que no ha significado que se haya suprimido acciones estatales como bien indican sus planes de Derechos Humanos para el reciente año pero si el abandono parcial de las temáticas LGBT si comparamos con el gobierno del PT. 


\section{El CASO DE ESPAÑa}

Vox es un partido político español fundado el 17 de diciembre de 2013 por Santiago Abascal. Nace del descontento de un sector conservador del Partido Popular (PP) ante las medidas centristas que adoptó Mariano Rajoy, a quien se le acusó de blando en su periodo de gobierno. La pretensión de este partido era ocupar el espacio electoral y político del PP y sustituirlo en la defensa de los valores clásicos de la derecha española. A ello, además, se le añadían reivindicaciones culturales, sociales y una mayor profundización en la liberalización de la economía española que la llevada durante el mandato de Mariano Rajoy (Gonzáles, 2019).

España, hasta hace pocos años, era uno de los países europeos en los que no había surgido un partido de derecha radical. Las razones que justificaron la excepción española fueron diversas: la excesiva vinculación que ha mantenido la extrema derecha española con el legado del régimen franquista, la relativa juventud del sistema democrático español, la no consolidación de la inmigración como un eje de confrontación política de primer orden y la monopolización del potencial espacio electoral de la derecha radical populista por parte del partido hegemónico de la derecha española, el Partido Popular (Hernández-Carr, 2011). Sin embargo, tras el surgimiento de Vox, España dejó de ser una excepción. El estudio de González (2019), basado en encuestas a expertos según la metodología de la Chapel Hill Expert Survey (2017), clasifica a Vox como de extrema derecha con 9,22 puntos sobre 10 y como un partido "muy cercano a posiciones tradicionalistas, autoritarias y nacionalistas" con un puntaje de 9,39 sobre 10 en la escala GAL/TAN (escala referida a los valores que ubica a los partidos en un espectro entre "verdes, alternativos y libertarios" y "tradicionalistas, autoritarios y nacionalistas") (González 2019: 54).

Las declaraciones del Secretario General de Vox, Javier Ortega Smith, a la cadena SER en una entrevista del 5 de diciembre del año 2018 ofrecen una estructura útil para explicar el ideario que el partido defiende. En dicha ocasión, Smith señaló que la "ideología" de Vox se resume en dos ideas: España y libertad. Cada una de ellas, evidentemente, evoca un concepto muy amplio que puede ser interpretado de diversas maneras. Es por ello que, sin abandonar la estructura propuesta por Smith, nos apoyamos en discursos de otros líderes de Vox para elaborar una síntesis de cómo el partido interpreta ambos conceptos que ha reconocido como centrales dentro de su ideario. En primer lugar, en cuanto a España, el partido enfatiza la unidad y la soberanía del país. La unidad de España se entiende tanto en términos de Estado como de nación. En términos de Estado, significa luchar contra todo intento de secesión territorial ${ }^{1} \mathrm{y}$, en el futuro, reemplazar el régimen de Comunidades Autónomas vigente desde el retorno a la democracia por un nuevo diseño de Estado unitario (Abascal, 17 de agosto de 2016). En términos de nación, la idea de unidad de España que defiende Vox es bastante más compleja. No es una idea directamente heredada del franquismo, en el sentido de una

\footnotetext{
${ }^{1}$ Consultado acerca de la posibilidad de un referéndum de secesión en Cataluña, Santiago Abascal señaló que su partido se opone a la realización de este tipo de referéndum, tanto si este se da solo en Cataluña como si se hiciese en todo el territorio español. El argumento que plantea para ello es que la generación actual de españoles no puede decidir acerca de la existencia misma de España, la cual se remonta a anteriores generaciones (Abascal, 17 de agosto de 2016).
} 
España homogénea en términos culturales ${ }^{2}$. Pero sí es una idea de España unida por costumbres y valores compartidos que trascienden las diferencias locales, así como por el orgullo que genera compartir un pasado glorioso. Existe en el partido una actitud nostálgica y una tendencia a idealizar algunos episodios de la historia, como por ejemplo la Reconquista y la Conquista de América ${ }^{3}$. Asimismo, Vox muestra un gran aprecio por modos de vida basados en la tradición, tales como la socialización en familias heterosexuales y extensas, la participación en festividades locales que mayormente coinciden con fechas clave del calendario litúrgico católico, así como algunas actividades de ocio relacionadas al mundo rural y no exentas de polémica como la caza y la tauromaquia. En cuanto a la soberanía, según Abascal (2016), esta debería residir en el «pueblo español»y no en las instituciones que pretenden representarlo, tales como los partidos políticos, los gobiernos de las Comunidades Autónomas o la Unión Europea. Respecto a esta última, Vox es bastante crítico de la pérdida de soberanía nacional que resulta de la integración europea, en particular en lo referente al control de las fronteras y la regulación de la inmigración.

En segundo lugar está el concepto de libertad. Este punto podría parecer contradictorio a la luz de lo que hemos señalado anteriormente, ya que sus ideas sobre la nación y la tradición hacen de Vox un partido fuertemente conservador. Sin embargo, el discurso del partido también apela frecuentemente al concepto de libertad individual, sin que por ello pueda ser calificado como un partido liberal. Vox aborda este concepto desde los ámbitos económico y social. En el aspecto económico, plantea la disminución de los impuestos y una intervención mínima del Estado que se justifica apelando a la libertad patrimonial. En el aspecto social, Vox plantea que la libertad consiste en que cada persona pueda formarse una manera propia de pensar y pueda expresarla sin necesariamente alinearse con lo políticamente correcto. En este punto, el partido muestra abiertamente su rechazo frente al llamado «consenso progre» (Abascal, 14 de abril de 2019), es decir, una manera de pensar progresista que, según Vox, ha sido establecida en estos tiempos por los partidos y grupos de izquierda como la única válida o como un ejemplo que se debería imitar. Desde Vox, además, se rechaza la colectivización de las personas bajo alguna etiqueta identitaria como el género o la orientación sexual. Así, en relación al tema de nuestro artículo, Vox defiende que las mujeres y las personas homosexuales no tienen por

\footnotetext{
${ }^{2}$ Esto se aprecia, por ejemplo, en el tema de la diversidad lingüística que caracteriza a España. En una intervención para la Fundación DENAES del año 2016, Santiago Abascal señala que su partido no se opone a las culturas y lenguas regionales, sino que plantea que existe una cultura española común, que está más allá de ellas y las unifica. Asimismo, el presidente de Vox señala que no busca prohibir las lenguas regionales; solo plantea que estas no deberían ser obligatorias para trabajar en la administración pública, ya que el español es la lengua que unifica a todos los ciudadanos (Abascal, 17 de agosto de 2016). Como vemos, lo que se plantea aquí no es reprimir o eliminar la diversidad, sino abarcarla dentro de un concepto mayor de identidad española.

${ }^{3}$ Excedería los propósitos de este artículo examinar el discurso de Vox referente a la historia española, así como algunas exageraciones e interpretaciones idealizadas en las que incurren los representantes del partido. Lo importante para un estudio de Vox en el aspecto político es entender que este tipo de discursos informan sus propuestas de política pública. Así, por ejemplo, la propuesta de una política migratoria mucho más benevolente con las personas hispanoamericanas que con las musulmanas (Abascal, 14 de abril de 2019) está respaldada por los discursos alusivos a los dos procesos históricos antes mencionados. Asimismo, la grandeza histórica de España es utilizada como argumento contra la Unión Europea, ya que se acusa a esta de haber relegado a la España heroica y conquistadora a un secundario rol de "camarera de Europa" (Abascal, 17 de agosto de 2016).
} 
qué seguir necesariamente el ideario de los movimientos internacionales del feminismo o de los colectivos LGTB.

\subsection{Revisión contextual sobre el feminismo en España}

Los primeros registros que se tiene del surgimiento del movimiento feminista en España datan de 1915, donde, con el establecimiento de la Asociación Nacional de Mujeres Españolas, las mujeres reclamaban una mayor participación en el ámbito laboral e igualdad de salarios. Sin embargo, fue con el establecimiento de la Segunda República en 1931 que el feminismo en este país alcanzó mayor visibilidad. Bajo el espíritu progresista de la Segunda República (1931 - 1939), se crearon las condiciones óptimas para mejorar la situación de las mujeres en la sociedad (Abella, 1996: 33). Ello se ve reflejado en la Constitución de 1931, la cual otorgó a las mujeres el derecho al seguro de maternidad, legalizó el matrimonio civil y también buscó erradicar la discriminación en los centros laborales; además, la libertad reproductiva de las mujeres fue salvaguardada mediante la legislación de anticoncepción (Martín Carretero, 1999: 14).

El gobierno del general Francisco Franco marcó el retroceso respecto a las expectativas de igualdad que se habían formado en la Segunda República (Ryan, 2006). Este choque ideológico entre la Segunda República y el franquismo (1939 - 1975) se ve reflejado en la interpretación que se empieza a dar de las políticas elaboradas en la época anterior. El progresismo que se defendió hasta 1939 fue interpretado por el gobierno de Franco como una muestra de la decadencia moral de la sociedad española. La alianza de este con la Iglesia Católica motivó a que se implementen legislaciones hostiles respecto a las políticas que incentivaron el progreso de las mujeres a nivel social, económico y personal; por lo tanto, un concepto esencialista y polarizado de las relaciones de género llegó a gobernar la vida española. En tal ambiente, los grupos feministas sufrieron un severo revés. Fueron considerados como grupos subversivos y se estableció una legislación estricta contra ellos. Como resultado, solo cuatro organizaciones feministas lograron obtener la aprobación oficial (Threlfall, 2004).

A poco tiempo de la muerte de Franco en 1975, España empezó a reorganizarse de un régimen autoritario a una democracia estable en poco tiempo. Las primeras elecciones generales fueron en 1977, la Constitución española se aprobó en 1978 y España ingresó a la Comunidad Económica Europea en 1986.

Para 1982, después de 5 años de gobierno bajo el mando de la Unión de Centro Democrático (UCD), un partido de centro derecha que desapareció con la transición democrática, el Partido Socialista Obrero Español (PSOE) lideró el gobierno español durante 14 años bajo la presidencia de Felipe González hasta 1996, cuando el conservador Partido Popular (PP) asumió el cargo con José María Aznar como gobernador por los siguientes ocho años. Luego, en 2004, el PSOE, bajo el liderazgo de José Luis Rodríguez Zapatero, gobernado nuevamente por casi otros ocho años. En noviembre de 2011, Mariano Rajoy (PP) ganó las elecciones generales con una mayoría absoluta en el parlamento nacional. 
Este rápido proceso de democratización, el cual tuvo lugar en menos de diez años (1975-1982), condujo a un profundo cambio social y con ello se incluyen los cambios de roles de las mujeres en la sociedad española. Para comprender la naturaleza de estos cambios debe señalarse que el estado de las mujeres en el régimen franquista (1939-1975) fue fuertemente influenciado por la Iglesia Católica y firmemente basada en el modelo de familia en donde el hombre ejercía un rol protector y las mujeres eran consideradas exclusivamente como amas de casa, cuyo único y principal papel era la maternidad (Bustelo, 2014). Esta perspectiva histórica aclara la importancia de cambio social y político y el impacto de las políticas de género en España en las últimas tres décadas. Una de las instituciones que resultó crucial para la visibilización del movimiento feminista en España fue el Instituto de la Mujer, creado en 1983 como organismo autónomo adscrito al Ministerio de Cultura y con el objetivo de "promover y fomentar las condiciones que posibiliten la igualdad social de ambos sexos y la participación de las mujeres en la vida política, cultural, económica y social" (Instituto de la Mujer y para la Igualdad de Oportunidades, s/f).

De acuerdo con Colomina, al volver al poder el PSOE bajo el liderazgo de Zapatero en el 2004, este se comprometió a deshacerse de la reputación de España como una sociedad ultraconservadora y dominada por los hombres. Este discurso se vio reflejado en las políticas de género que trajeron más cambios en la legislación de igualdad. El gobierno del PSOE desarrolló una estrategia para promover la igualdad de género a través de un gobierno de paridad y un aumento en la representación política de las mujeres (Colomina, 2014: 9). Sumado a ello, se dio la reforma del Código Civil para permitir el matrimonio homosexual, la aprobación de la Ley de Igualdad Efectiva, la reforma de la legislación sobre el aborto y la reforma del régimen de seguridad social para las trabajadoras domésticas (Bustelo, 2014). Con la llegada al poder de Mariano Rajoy -líder del Partido Popular- en el año 2011, se retrajeron algunas medidas que habían sido adoptadas durante el gobierno de Zapatero. En este periodo, la paridad en el gobierno desapareció y la proporción de mujeres en el gabinete del PP cayó al 31\% (Bustelo, 2014). Asimismo, el Instituto de la Mujer fue degradado jerárquicamente (Bustelo, 2014).

Al mismo tiempo en el que se desarrollaban estos cambios económicos y políticos, un nuevo partido político surgiría: Podemos. Este partido, liderado por Pablo Iglesias y situado en la izquierda del espectro político, tuvo su origen en una serie de movimientos sociales diversos y canaliza el vacío de representación dejado por el PSOE en la población española luego de la crisis económica. Respondiendo al hecho de que los movimientos feministas fueron cercanos al partido desde su origen, uno de los pilares importantes que defiende Podemos es la defensa de la equidad de género, a tal punto que entre sus propuestas económicas encontramos la "economía feminista" que se sustenta, entre otros aspectos, en una visión más colaborativa y menos individual del desarrollo económico y en un reparto más equitativo del trabajo de cuidado entre hombres y mujeres -incluyendo, por ejemplo, permisos de maternidad y paternidad de igual duración, y un sistema público de atención a las personas dependientes para evitar que sean las mujeres de sus familias quienes se vean sobrecargadas con la responsabilidad de cuidar de ellas (Requena, 2015). 


\subsection{Revisión contextual sobre el feminismo en España}

En el caso de Vox, sus ideas referentes al feminismo se desprenden del ideario general del partido que hemos presentado en la primera sección. Así, uno de los aspectos centrales en el discurso de Vox sobre las mujeres es que ellas no deben ser colectivizadas -es decir, agrupadas bajo un concepto de lo que debe ser "la mujer"- ni presionadas para seguir la línea ideológica prevaleciente en el movimiento feminista a nivel internacional. Por el contrario, ellas deben ser libres para pensar y actuar según les parezca. La oposición de Vox en contra del llamado "feminismo supremacista" -un término que suele aparecer en el discurso de Rocío Monasterio, la mujer más visible del partido- se enmarca dentro de la oposición general en contra de lo políticamente correcto o el "pensamiento progre" -un término al que ya hemos aludido anteriormente y que suele ser usado por Santiago Abascal.

En el caso del feminismo, Vox plantea que este está siendo entendido actualmente de una manera que no favorece a la mujer, sino que más bien la presenta como una víctima o como una persona desfavorecida a la cual el Estado debe ayudar. Un ejemplo que reflejó de manera muy clara lo que acabamos de señalar fue el vídeo publicado por Rocío Monasterio, actualmente presidenta del partido en la Comunidad de Madrid, con motivo del Día Internacional de la Mujer. Como representante de Vox, Rocío Monasterio mantuvo un discurso que buscaba romper con la visión del feminismo como el medio que permite mejorar las condiciones de las mujeres. En lugar de ello, planteó la imagen de una mujer que no necesita apoyo alguno para sobresalir en la sociedad. Al contrario, dio a entender que si se brinda algún apoyo adicional a las mujeres solo por el hecho de serlo, entonces se estaría cayendo en una mirada paternalista o condescendiente hacia ellas. En dicho video, ella aparece acompañada de varias mujeres de su partido y señala lo siguiente:

No hables en mi nombre porque soy mujer y no soy víctima por el hecho de nacer mujer. Soy mujer y el Estado no me tiene que proteger, ni dar, ni quitar. Tengo los mismos derechos que el hombre por ser española. Soy mujer y no seré dócil, ni sumisa, ni callada ante el feminismo supremacista. Soy mujer y denuncio a los que nos queréis colectivizar. Soy mujer y no pretendo que todas las mujeres pensemos igual. [...] Soy mujer y no necesito que el Estado me garantice mi puesto de trabajo con cuotas. Ya me lo gano yo. No necesito la condescendencia del hombre ni de las feministas supremacistas (Monasterio, 4 de marzo de 2019).

A lo largo del video, y en los carteles que las participantes llevaban, se repite la frase "No hables en mi nombre". Ello da cuenta de la insistencia de Vox en evitar que las mujeres sean colectivizadas y que quienes forman parte de organizaciones feministas se expresen a nombre de todo el conjunto de mujeres. En el discurso de Vox es muy importante señalar que hay mujeres que no apoyan las ideas y prácticas actuales del movimiento feminista y que ellas también deben ser tomadas en cuenta. Esto lo resalta Rocío Monasterio cuando aclara públicamente que ella no participará en la huelga de mujeres convocada para el 8 de marzo (Monasterio, 7 de marzo de 2019). Asimismo, es importante notar que Vox sí reconoce los logros históricos del movimiento feminista en España, sobre todo en cuestiones como la educación, el voto o la posibilidad de trabajar. 
Pero está en contra de aquello que percibe como una deriva extrema o "supremacista" que el movimiento ha tomado en la actualidad.

Con el mismo motivo de conmemorar el Día Internacional de la Mujer, la cadena SER convocó a un debate entre cinco mujeres que participaban activamente en la política española, entre ellas Rocío Monasterio, quien acudía en representación de Vox. Respecto al feminismo, ella declaró lo siguiente:

Para nosotros el feminismo es la defensa de los derechos, de la igualdad entre los hombres y las mujeres. Y estamos orgullosos de ese feminismo español, de Campoamor, de Pardo Bazán, de Concepción Arenal, que tanto lucharon por la educación [...] o que tanto lucharon por que tuviéramos acceso a la autonomía económica o que tuviéramos derecho al voto. Nosotros de ese feminismo sí que estamos orgullosos, pero denunciamos el feminismo supremacista que hoy quiere amordazar a algunas mujeres que discrepan de la doctrina que nos quieren imponer otros y denunciamos que se nos intente colectivizar a las mujeres y denunciamos que los partidos piensen que son propietarios del pensamiento de las mujeres. Nosotros queremos que cada mujer haga lo que le dé la gana, que tenga libertad (Monasterio, 7 de marzo de 2019).

Otro tema en el que Vox ha mostrado su oposición contra la deriva contemporánea del feminismo es el uso del lenguaje inclusivo. Irene Montero, portavoz de Unidas Podemos, señaló en una sesión del Congreso de los Diputados que "El lenguaje se utiliza como instrumento para perpetuar el machismo en las sociedades. Lo que no se nombra suele no existir y es muy curioso que la mayoría de los lenguajes utilicen el masculino para referirse también a la otra mitad de la población que somos las mujeres" (Montero, 8 de febrero de 2018). Con ello, pretendió mostrar que existe una invisibilización de las mujeres en el plano lingüístico. Es más, el mismo hecho de que su partido haya adoptado el nombre de Unidas Podemos luego de su alianza con Izquierda Unida para las elecciones generales y europeas de 2019 indica que existe, al menos en el discurso, esta preocupación por reivindicar el género femenino en el lenguaje. Como reacción a esta postura, Rocío Monasterio ha evitado la feminización del nombre de su profesión y de los cargos que actualmente asume. Se presenta a sí misma como "arquitecto" y "Presidente de Vox Madrid". En una entrevista, señaló que hace esto como "una forma de rechazar la ridícula imposición de la ideología de género y el absurdo del lenguaje inclusivo" (Monasterio, 24 de marzo de 2019).

Continuando con los temas referentes al lenguaje, Vox evita usar la palabra "género" y, bajo su concepción de igualdad, evita también considerar al género como una categoría relevante para el análisis de temas como la representación política o la violencia. Para Vox, las llamadas cuotas de género son una forma de discriminación por motivo de sexo. En su programa, respecto a la Ley Electoral, el partido propone realizar una «Supresión de cuotas por sexo o por cualquier otra causa en las listas electorales. Cada partido las elaborará de acuerdo a las decisiones de sus militantes» (Punto 12). Esto es consecuente también con la idea de que las mujeres no necesitan la ayuda de las cuotas para llegar al poder, sino que pueden conseguirlo por sus propios méritos.

Por otro lado, el partido incluye la violencia contra las mujeres dentro del concepto amplio de "violencia intrafamiliar" y se rehúsa a emplear los términos "violencia 
machista" o "violencia de género". En el apartado "Vida y Familia", propone la «Derogación Ley de Violencia de Género y de toda norma que discrimine a un sexo de otro. En su lugar, promulgar una ley de violencia intrafamiliar que proteja por igual a ancianos, hombres, mujeres y niños. Supresión de organismos feministas radicales subvencionados, persecución efectiva de denuncias falsas. Protección del menor en los procesos de divorcio» (Punto 70). En la práctica, a pesar de no formar parte de un gobierno nacional, Vox aprovecha eventos conmemorativos y espacios públicos para defender su postura favorable a la derogación de dicha ley. Así, por ejemplo, el 25 de noviembre de 2019 -Día Internacional de la Eliminación de la Violencia contra la MujerJavier Ortega Smith protagonizó un polémico incidente en el Ayuntamiento de Madrid debido a que sus declaraciones fueron consideradas irrespetuosas por varias mujeres asistentes al evento y generaron también el rechazo del alcalde perteneciente al PP (Roces y Belver, 2019).

En todos estos ejemplos vemos que Vox recurre a un discurso abiertamente cuestionador del feminismo; sin embargo, también es notorio que se concentra en criticar al feminismo actual mientras que reconoce e incluso admite cierta admiración por el histórico. Asimismo, plantea una concepción bastante básica de igualdad entre hombres y mujeres -prácticamente reducida al ámbito de la igualdad ante la ley- y ajena a las discusiones contemporáneas sobre las brechas de género. En este sentido, concluimos para el caso español que el discurso de Vox no es completamente un discurso antifeminista, sino que sería más apropiado entenderlo como una reacción contra los valores postmateriales representados por los partidos más progresistas, en particular por los de izquierda. A continuación ahondamos un poco más en la explicación de los valores materiales y post-materiales.

Inglehart ya hacía referencia a un cambio en los valores culturales de las sociedades en dos ejes principales: un primer eje compuesto por valores de supervivencia típicos de sociedades poco desarrolladas económicamente en las que la seguridad personal no está garantizada para la mayor parte de los individuos, y un segundo eje en el que las sociedades transitan hacia un sistema de valores secular-racionales o postmateriales que incluyen, por ejemplo, la mayor valoración de la satisfacción personal y la autoexpresión, la prioridad de la conservación del medio ambiente, el apoyo a la comunidad LGTB y la igualdad entre hombres y mujeres entendida como una igualdad efectiva y no solo formal (Díez, 2011: 11).

En Europa, el surgimiento de los Estados de Bienestar contribuyó a la adopción de valores post-materiales. Entonces se produjo un declive en las tensiones políticas tradicionales de carácter económico. Las nuevas generaciones, educadas en el contexto de la abundancia, cambiaron su sistema de valores. A diferencia de la generación de la posguerra, las nuevas generaciones empezaron a incorporar más aspectos no económicos dentro de sus nociones de calidad de vida y búsqueda de la realización personal. Actualmente, la centralidad de las demandas materiales ha vuelto a surgir de una nueva manera. La crisis económica trajo de nuevo al centro de la discusión temas como la pobreza, la precariedad del empleo, las dificultades para emanciparse y acceder a una 
vivienda digna, entre otros. Asimismo, el desafío de la migración hacia Europa trajo consigo preocupaciones por la seguridad personal de los ciudadanos. Como resultado de todo ello, tenemos ahora un encuentro bastante tenso entre propuestas políticas que pretenden responder a este tipo de desafíos de una manera más post-material -como las de Unidas Podemos- y de una manera más material y tradicional -como las de Vox.

Discursivamente, la tensión que se genera entre Vox y los partidos progresistas gira en torno a los valores post-materiales y no a la defensa de un antifeminismo en sí. Es por ello que, desde la perspectiva de los representantes de Vox que hemos citado aquí, hay logros feministas dignos de reconocimiento, y son precisamente aquellos que se vinculan con la seguridad material de las mujeres -como estudiar para luego poder trabajar y ganarse la vida. Por el contrario, otros asuntos como el lenguaje inclusivo o la huelga del 8 de marzo son vistos como exageraciones o frivolidades del feminismo contemporáneo. Asimismo, de manera consecuente con su pensamiento favorable a una intervención mínima del Estado, Vox le asigna al Estado un papel de poco alcance en cuanto a la garantía de igualdad entre hombres y mujeres. Se limita a la igualdad formal que aparece en la Constitución y rechaza los intentos de profundizar hacia una igualdad más efectiva. En este punto, Vox llega incluso a proponer un retroceso en las competencias del Estado cuando afirma, en el punto 56 de su programa, que el financiamiento de la sanidad pública debería ser más austero y no cubrir intervenciones "ajenas a la salud" como el aborto, que actualmente es legal en España.

\section{Conclusiones}

En este artículo hemos abordado la influencia del antifeminismo en los discursos políticos de Vox en España y de Jair Bolsonaro en Brasil, ambos considerados como de extrema derecha. Antes de compararlos, reconocemos las divergencias entre nuestros casos en tres puntos primordiales: el tipo de actor, el acceso al poder y el sistema político del país. En el caso del Vox, nos referimos a un partido político que, si bien cuenta con 52 representantes en el parlamento, aún no ha logrado formar un gobierno nacional, mientras que Jair Bolsonaro ha llegado a la presidencia de Brasil acompañado de un partido que es, sobre todo, un grupo de personajes conservadores reunidos en torno a la figura del candidato. El otro punto fundamental es el sistema político, ya que el sistema presidencialista de Brasil reduce la dependencia frente a la necesidad de coaliciones en el Legislativo. Pese a ello, encontramos útil la comparación de estos casos por el hecho de que ambos son fenómenos políticos de reacción ante los avances progresistas de sus gobiernos predecesores.

Como elementos comunes en el contexto en que ambos operan, identificamos un avance de gobiernos previos en favor de la libertad de las mujeres, así como la politización de problemas como el aborto, la inequidad entre hombres y mujeres o la violencia de género. En esto último, han tenido un rol importante no solo los gobiernos progresistas, sino también los movimientos sociales. Actualmente, ambas candidaturas que estudiamos cuestionan, e incluso buscan revertir hasta cierto punto, dichos avances. Hay en ambos una crítica al feminismo actual. Ambos aprecian los logros históricos del movimiento -en especial en el plano económico como el acceso al empleo o la lucha por 
iguales salarios. Sin embargo, consideran que el feminismo contemporáneo ha tomado una deriva exagerada en las formas y se está volviendo contraproducente en el fondo. Respecto a las formas, recordemos la frase de Alves sobre las mujeres que protestan mostrando los senos o las declaraciones de Monasterio sobre la inutilidad del lenguaje inclusivo. Respecto al fondo, ambos plantean que el feminismo se está desviando de su propósito original y se está volviendo una especie de batalla de las mujeres contra los hombres en la que ya no se defiende a todas las personas por igual. Esto es notorio en el discurso que ambas candidaturas manejan acerca de la violencia contra las mujeres, a la cual evitan llamar "violencia de género". Asimismo, critican que el feminismo actual está dejando de representar a las mujeres conservadoras, ya que se muestra como decididamente progresista y políticamente correcto.

La diferencia principal, sin embargo, está en el nivel de debate y crítica al desarrollo previo de políticas públicas influenciadas por el feminismo. La compresión de la teoría de género y los límites que se consideran aceptables en el discurso político son mayores en el caso de España, donde se está debatiendo temas más complejos y que siguen en discusión incluso dentro del mismo movimiento feminista, como las cuotas de género en la representación política. El debate está menos desarrollado en Brasil y, por lo tanto, se problematizan temas más elementales como los roles de género -expresados en la frase "los niños visten de azul y las niñas de rosa"- y el papel de la Iglesia como baluarte y guía moral. Asimismo, en Brasil, el rechazo contra las políticas de género se encuentra enmarcado dentro de un discurso de oposición y culpabilización en contra de prácticamente todo aquello que provenga del PT. En el caso del Vox, en cambio, si bien existe oposición, no se trata solamente de contradecir al PSOE o a Unidas Podemos, sino de un cuestionamiento más general al progresismo de la sociedad actual. Luego de realizar esta investigación, hemos encontrado en nuestros casos de estudio -y de manera más evidente y mejor construida en el caso de Vox- una crítica válida hacia cómo se está tratando el tema de género en la actualidad y cuál es el rol de los Estados en ello.

Finalmente, mirando hacia el futuro, podemos encontrar que efectivamente existe un desfase entre el discurso como como candidato y como gobierno, pues existen trabas reales para que los líderes que hemos estudiado puedan llevar a cabo cualquier acción pública, más aún cuando existe una oposición considerable y organizada. En este sentido, es probable que si hubiese un gobierno nacional en el que Vox participase, este encontraría obstáculos similares -o incluso más fuertes dada la naturaleza del sistema político español- a los que encontró el partido de Bolsonaro con la ministra Alves al frente. Es posible que este desfase que identificamos en el artículo entre los discursos de Bolsonaro y los de Alves, y entre estos dos discursos y los hechos, sería también replicado en el caso español. 


\section{REFERENCIAS}

Abascal, S. (2016, 17 de agosto). Los partidos políticos y la Nación española. Lección para la X Escuela de Verano de la Fundación DENAES [Archivo de video]. Recuperado de https://bit.ly/2TgObHm

Abascal, S. (2019, 14 de abril). Santiago Abascal y Morante de la Puebla. Paseíllo al natural [Archivo de video]. Recuperado de https://bit.ly/394sn8G

Abella, R. (1996). La vida amorosa en la Segunda República. Madrid: Ediciones Tema de Hoy.

Alves, D. (2019, 3 de enero). Damares Alves: menino veste azul [Archivo de video]. Recuperado de https://bit.ly/2T2bohx

Alves, D. [@damaresalves]. (2019, 22 de enero). Recuperado de https://bit.ly/32zQHN5

Alves, D. [@damaresalves]. (2019, 29 de enero). Recuperado de https://bit.ly/2PwbFHA

Alves, D. [@damaresalves]. (2019, 10 de marzo). Recuperado de https://bit.ly/3cccM96

Alves, D. [@damaresalves]. (2019, 11 de marzo). Recuperado de https://bit.ly/3859HnT

Alves, D. [@damaresalves]. (2019, 12 de marzo). Recuperado de https://bit.ly/2T2FzoT

Alves, D. [@damaresalves]. (2019, 13 de marzo). Recuperado de https://bit.ly/2uE9j28

Alves, D. [@damaresalves]. (2019, 17 de marzo). Recuperado de https://bit.ly/32FZspb

Alves, D. [@damaresalves]. (2019, 20 de marzo). Recuperado de https://bit.ly/32vgLc7

Alves, D. [@damaresalves]. (2019, 25 de abril). Recuperado de https://bit.ly/2Tm2CK6

Alves, D. [@damaresalves]. (2019, 2 de mayo). Recuperado de https://bit.ly/2VpryDp

Alves, D. [@damaresalves]. (2019, 4 de mayo). Recuperado de https://bit.ly/2HZMLfm

Alves, D. [@damaresalves]. (2019, 29 de junio). Recuperado de https://bit.ly/2vnfI1M

Barbiéri, F (2019, 16 de abril). Damares diz que na 'concepção cristã' mulher deve ser 'submissa' ao homem no casamento. O Globo [Archivo de video] Recuperado de https://glo.bo/2VsGiNp

Blay, E. A. (1987). "O visível e o limite dos movimentos sociais na construção da prática pública”. En E. M. Oliveira (org.). Mulheres: da domesticidade à cidadania. Estudos sobre movimentos sociais e democratização. Águas de São Pedro, SP: ANPOCS, Conselho Nacional dos Direitos da Mulher.

Bustelo, M. (2014). Three Decades of State Feminism and Gender Equality Policies in Multi-governed Spain. Sex Roles, 74 (3 - 4), 107 - 120.

Borba, A. (1998). "Legislando para as mulheres". En Borba, A.; Faria, N. y Godinho, T. (orgs.). Mulher e política: gênero e feminismo no Partido dos Trabalhadores. Sao Paulo: Fundación Perceu Abramo.

Colomina, I. (2014). Women, Transition and Social Changes. The case of Spain, 19761986. En Women, Leadership and Society. Conferencia llevada a cabo en Yerevan, Armenia.

Díez, J. (2011). ¿Regreso a los valores materialistas? El dilema entre seguridad y libertad en los países desarrollados. Revista Española de Sociología, 9 - 46.

Fukuyama, F. (2018) Identity: The Demand for Dignity and the Politics of Resentment. Nueva York: Farrar, Straus y Giroux.

González, P. (2019). Mapeando a Vox en el espacio político multidimensional: un estudio comparado de escalas y dimensiones. (Trabajo de Fin de Grado). Universidad de Salamanca.

Grugel, J. y Riggirozzi, P. (2018). Neoliberal disruption and neoliberalism's afterlife in Latin America: What is left of post-neoliberalism? Critical Social Policy, 38 (3), 547-566.

Hernández-Carr, A. (2011) La derecha radical populista en Europa: discurso, electorado y explicaciones. Reis, 136, 141-160. 
Inglehart, Ronald y Pippa Norris (2017). Trump and the Populist Authoritarian Parties: The Silent Revolution in Reverse. Perspectives on Politics, 15(2), 443-454.

Instituto de la Mujer y para la Igualdad de Oportunidades (s/f) Historia: Nace el Instituto de la Mujer. Recuperado de: https://bit.ly/2vk8w6K

Kahhat, F. (2019). El eterno retorno. La derecha radical en el mundo contemporáneo. Lima: Editorial Crítica.

Levitsky, S. y Roberts, K. M. (2011). The resurgence of the Latin American left. Baltimore: Johns Hopkins University Press.

Suely, T. y Brabo, M. (2015) Igualdad de género en Brasil. Revista de Trabajo Social UNAM, 7(10), 103-126.

Mariano, R. y Gerardi, D. A. (2019). Eleições presidenciais na América Latina em 2018 e ativismo político de evangélicos conservadores. Revista USP, (120), 61-76. https://doi.org/10.11606/issn.2316-9036.v0i120p61-76

Martin Carretero, C. (1999). Las mujeres jóvenes: empleo, educación y familia. Avances y retrocesos en la igualdad de oportunidades. Madrid: Instituto de la Juventud.

Ministério da Mulher, da Família e dos Direitos Humanos [@ Direitos Humanos Brasil] (2019, 2 de enero). O Ministério da Mulher, da Família e dos Direitos Humanos promoveu uma Cerimônia de Boas-Vindas nesta quarta-feira (02), em Brasília, para a posse da ministra Damares Alves. [Post en Facebook]. Recuperado de https://bit.ly/2I7pRCQ

Molyneaux, M. (2003). Movimientos de mujeres en América Latina. Estudio teórico comparado. Madrid: Cátedra, Universidad de Valência, Instituto de la Mujer

Monasterio, R (2019, 4 de marzo) Las mujeres de VOX rompen con la huelga feminista del 8M [Archivo de vídeo]. Recuperado de https://bit.ly/3ahGzLK

Monasterio, R (2019, 7 de marzo) Debate sobre feminismo y mujer con políticas españolas. Cadena SER. [Archivo de vídeo]. Recuperado de_https://bit.ly/39njF5i

Monasterio, R. (2019, 24 de marzo). Monasterio pasa del lenguaje inclusivo y utiliza el masculino en sus cargos de Vox. [Archivo de vídeo]. Recuperado de https://bit.ly/3ciRs1r

Montero, I (2018, 8 de febrero) Montero defiende "portavoza" para dar visibilidad a la mujer. Europa Press [Archivo de vídeo]. Recuperado de_https://bit.ly/2PBCav3

Mudde, C. y Rovira Kaltwasser, C. (2017). Populism: A Very Short Introduction. Oxford: Oxford University Press.

Norris, P. y R. Inglehart (2019). Cultural Backlash. Trump, Brexit, and Authoritarian populism. Cambridge: Cambridge University Press.

O Globo. (2019, 3 de enero). Confira a íntegra do pronunciamento do presidente Jair Bolsonaro. Brasilia. Recuperado de https://glo.bo/2PAIROe

Ohara, C. (2019). Posso ir às ruas de braços dados com feministas por salários iguais, diz Damares. Folha de Sao Paulo, 18 de febrero. Recuperado de https://bit.ly/2TpajPP

Ortega Smith, J (2018, 5 de diciembre) Entrevista. Vox carga contra las "feminazis" y niega que exista la violencia machista [Archivo de video]. Recuperado de https://bit.ly/3chVzuM

Palermo, V y Menezes, T (2013). Lulismo, gobierno de Lula y transformaciones de la sociedad brasileña: los términos del debate interpretativo. Miríada, 5(9), 21-66.

Requena, A. (2015) Claves de la propuesta de economía feminista que Podemos tiene sobre la mesa. El Diario, 2 de marzo. Recuperado de https://bit.ly/3a8oAY2

Roces, P. y Belver, M. (2019) Mujeres abandonan el acto contra la violencia de género en el Ayuntamiento de Madrid durante el discurso de Ortega Smith. El Mundo, 25 de noviembre. Recuperado de https://bit.ly/387gH3z 
Ryan, L. (2006) “A Case Apart: The Evolution of Spanish Feminism”. En Perlan, R. (ed.) Feminisms Within and without. Galway: National Women Studies Centre.

Threlfall, Monica. (2004). "Gendering the Transition to Democracy: Reassessing the Impact of Women's Activism" En Cousins, C.; Threlfall, M. y Valiente, C. (eds.) Gendering Spanish Democracy. Londres y Nueva York: Routledge.

Taguieff, P.A. (2007). "Interpretar la ola populista en la Europa contemporánea: entre resurgencia y emergencia”. En Simón, M.A. La extrema derecha en Europa desde 1945 a nuestros días. Madrid: Tecnos.

Vox (2018) 100 medidas para la España viva. Recuperado de https://bit.ly/2wjHcG0 